\title{
Structure of manganese zinc ferrite spinel nanoparticles prepared with co-precipitation in reversed microemulsions
}

\author{
Darko Makovec · Alojz Kodre · Iztok Arčon • \\ Miha Drofenik
}

Received: 12 February 2008/ Accepted: 31 August 2008

(C) Springer Science+Business Media B.V. 2008

\begin{abstract}
The structure of $\mathrm{Mn}_{0.5} \mathrm{Zn}_{0.5} \mathrm{Fe}_{2} \mathrm{O}_{4}$ spinel ferrite nanoparticles is studied as a function of their size and the experimental conditions of their synthesis using X-ray absorption spectroscopy. The nanoparticles of different sizes down to approximately $2 \mathrm{~nm}$ and with a narrow size distribution were synthesized using co-precipitation in reverse microemulsions. Simultaneous refinement of the X-ray absorption fine structure (EXAFS) of three constituting metals shows a migration of $\mathrm{Mn}$ and $\mathrm{Zn}$ ions to the octahedral site of the spinel lattice compensated by the corresponding migration of the Fe ions. To a smaller extent, Mn ions switch the occupation site already in bulk and in larger nanoparticles, while a
\end{abstract}

Electronic supplementary material The online version of this article (doi:10.1007/s11051-008-9510-0) contains supplementary material, which is available to authorized users.

D. Makovec $(\square)$ · A. Kodre · I. Arčon · M. Drofenik Jožef Stefan Institute, Ljubljana, Slovenia

e-mail: Darko.Makovec@ijs.si

\section{A. Kodre}

Faculty of Mathematics and Physics, University of Ljubljana, Ljubljana, Slovenia

I. Arčon

University of Nova Gorica, Nova Gorica,

Slovenia

M. Drofenik

Faculty of Chemistry and Chemical Engineering,

University of Maribor, Maribor, Slovenia sporadic migration of $\mathrm{Zn}$ is detected only in the nanoparticles with sizes below approximately $5 \mathrm{~nm}$. $\mathrm{X}$-ray absorption near edge structure (XANES) reveals considerable variations in the position of the Mn $K$ edge, suggesting the average Mn valence in the nanoparticles to be higher than 3+. Annealing at $500{ }^{\circ} \mathrm{C}$ relaxes the structure of as-synthesized nanoparticles toward the structure of the ceramic bulk standard.

Keywords Nanoparticles - Spinel ferrites · Structure - EXAFS · Magnetic properties . Nanomaterial

\section{Introduction}

Magnetic properties of nanoparticles have been intensively studied, and the mechanism of the size effect is generally well understood in terms of the magnetically inactive surface layer (Kodama 1999; Batlle and Labarta 2002). In the mixed-oxide nanoparticles, the magnetization may additionally be affected through the influence of the small size on the structure of nanoparticles: the adaptation to the small size and the large surface-to-volume ratio. The flexibility of the crystal structure is particularly pronounced in ferrite spinel nanoparticles.

The spinel $\mathrm{AB}_{2} \mathrm{O}_{4}$ structure can be generally described as a cubic close-pack arrangement of oxygen ions in which tetrahedral A and octahedral 
B interstitial lattice sites are occupied by cations. In the normal spinel, the tetrahedral sites are occupied by divalent cations while trivalent cations occupy octahedral sites. In contrast, 2-valent cations occupy octahedral sites in inverse spinel, whereas 3-valent cations are distributed equally among A- and B-sites. In ferrites, $\mathrm{Fe}^{3+}$ serves as the trivalent cation. Generally, the structure of spinel ferrites could be described with the $\left(\mathrm{Me}_{1}^{2+}{ }_{X} \mathrm{Fe}_{X}^{3+}\right)\left[\mathrm{Me}_{X}^{2+} \mathrm{Fe}_{2-}^{3+}\right] \mathrm{O}_{4}$ formula, where round and square brackets represent tetrahedral and octahedral sites, respectively. The degree of inversion $X$ is the proportion of the trivalent cation $\mathrm{Fe}^{3+}$ occupying tetrahedral sites (Smit and Wijn 1959).

The magnetic properties of the spinel ferrite materials originate from the antiferromagnetic coupling between the octahedral and tetrahedral sublattices. The magnetization results from the difference between the magnetizations of $\mathrm{A}-$ and B-sublattice and is thus directly related to the population of the sublattices with different cations. In the bulk material, the majority of divalent cations $\left(\mathrm{Fe}^{2+}\right.$, $\mathrm{Co}^{2+}, \mathrm{Ni}^{2+}, \mathrm{Cu}^{2+}, \mathrm{Mg}^{2+}$ ) prefers incorporation at the larger octahedral lattice sites. In contrast, only $\mathrm{Zn}^{2+}$ shows almost total preference to occupy the tetrahedral sites. Diamagnetic $\mathrm{Zn}^{2+}$ is usually used in mixed ferrites to decrease the magnetic moment of the A-sublattice, thus increasing the overall magnetization of the crystal. Pure $\mathrm{ZnFe}_{2} \mathrm{O}_{4}$ bulk material is paramagnetic at room temperature, since it has the normal spinel structure with $\mathrm{Zn}$ incorporated almost exclusively at the tetrahedral lattice sites; there is no superexchange interaction between the two sublattices, which would result in ferrimagnetism. Another divalent ion, which displays preference for the tetrahedral A-sites, is $\mathrm{Mn}^{2+}$. In pure $\mathrm{MnFe}_{2} \mathrm{O}_{4}$ ceramics, approximately $80 \%$ of $\mathrm{Mn}$ enters the spinel structure at tetrahedral sites and $20 \%$ at octahedral sites: $\left(\mathrm{Mn}_{0.8} \mathrm{Fe}_{0.2}\right)\left[\mathrm{Mn}_{0.2} \mathrm{Fe}_{0.8}\right] \mathrm{O}_{4}$ (Smit and Wijn 1959).

The structure of the nanoparticles has been shown to differ significantly from that of the corresponding bulk materials. For example, $\mathrm{ZnFe}_{2} \mathrm{O}_{4}$ becomes ferrimagnetic, when prepared in the form of nanoparticles, because it adopts a partially inverted spinel structure (Sato et al. 1990; Kamiyama et al. 1992; Jeyadevan et al. 1994; Hamdeh et al. 1997; Ammar et al. 2004). Using neutron powder diffraction, the degree of inversion $X$ for $\mathrm{ZnFe}_{2} \mathrm{O}_{4}$ nanoparticles prepared by co-precipitation was determined to increase with decreasing particle size from 0.108 at particle size $9.6 \mathrm{~nm}$ to 0.142 at particle size $2.9 \mathrm{~nm}$ (Kamiyama et al. 1992). Besides on the particle size, the degree of inversion depends also on the preparation method (Hamdeh et al. 1997; Ammar et al. 2004). A higher degree of inversion in nanoparticles than in the bulk material was also detected in $\mathrm{MnFe}_{2} \mathrm{O}_{4}$ (Chen et al. 1996; Carpenter et al. 1999) and in mixed MnZn ferrites (Fetami et al. 1998; Jeyadevan et al. 2000; Calvin et al. 2002a). Calvin et al. (2002b) used multiedge refinement of extended X-ray absorption fine structure (EXAFS) of MnZnferrite nanoparticles to quantitatively determine the population of both sublattices of the spinel structure by constituting cations. The nanoparticles with sizes ranging from $\sim 11$ to $\sim 15 \mathrm{~nm}$ were synthesized with calcination of the hydroxide precursors at temperatures around $500{ }^{\circ} \mathrm{C}$. Even the samples prepared under very similar conditions exhibited remarkable differences in the cation distribution. Nearly $100 \%$ of $\mathrm{Zn}$ was incorporated at tetrahedral lattice sites, while the proportion of $\mathrm{Mn}$ and $\mathrm{Fe}$ at tetrahedral sites varied considerably between $14 \%$ and $50 \%$ for $\mathrm{Mn}$ and between $17 \%$ and $27 \%$ for $\mathrm{Fe}$.

The structure of the ferrite nanoparticles with sizes above $\sim 10 \mathrm{~nm}$, studied so far, is sufficiently close to the structure of the bulk spinel so that ceramic standards have been used to calibrate some parameters of the structure model.

In the present study, the structure of even smaller MnZn-ferrite nanoparticles with sizes down to $\sim 2 \mathrm{~nm}$ has been investigated. The nanoparticles are synthesized using co-precipitation in reverse microemulsions, and the size, with a narrow distribution, is determined by varying the experimental conditions. The main goal of the study is to find whether the decrease in particle size is accompanied by any systematic structural change.

\section{Experimental}

Synthesis of nanoparticles

MnZn-ferrite nanoparticles were synthesized using co-precipitation in the environment of the inverse microemulsions. The microemulsion method has been shown to be a very promising laboratory technique for the preparation of a small quantity of nanoparticles, 
providing an excellent control over the particles' size and shape, as well as the homogeneity and the chemical composition. The co-precipitation occurs in reverse micelles - tiny droplets of aqueous phase that are embedded with a surfactant in an oil phase. The water pools of the reverse micelles act as nanoreactors for the synthesis of the particles. The particle size of the product depends primarily on the size of the pools and on the concentration of the reactants dissolved in the aqueous phase. The volume of the reverse micelles is thermodynamically determined, by the water-tosurfactant molar ratio $w$ and the temperature. Thus, the size of the nanoparticles can be controlled by varying the microemulsion composition, the concentration of reactants, or the temperature (Pileni 1993; Makovec et al. 2004; Košak et al. 2005).

In this work, the MnZn-ferrite nanoparticles were synthesized using the co-precipitation of hydroxides of $\mathrm{Mn}^{2+}, \mathrm{Zn}^{2+}$, and $\mathrm{Fe}^{2+}$ ions in a microemulsion system consisting of an aqueous phase, $n$-hexanol as the oil phase, and $n$-hexadecil trimethylammonium bromide (CTAB) as the surfactant. The oxidation of the $\mathrm{Fe}(\mathrm{II})$ hydroxide during the aging of the precipitates in air resulted in a direct formation of the MnZn ferrite spinel. The details of the synthesis method are described in Makovec et al. 2004. The co-precipitation of the hydroxides from the microemulsion containing metal ions (Microemulsion I) was effected by admixing an appropriate amount of the Microemulsion II containing the precipitation agent tetramethyl ammonium hydroxide (TMAH). The aqueous phase of the Microemulsion I was prepared by dissolving metal sulfates $\left(\mathrm{FeSO}_{4}, \mathrm{ZnSO}_{4}, \mathrm{MnSO}_{4}\right.$ in the molar ratio $0.5: 0.5: 2$ ) in diluted $\mathrm{H}_{2} \mathrm{SO}_{4}$ at $\mathrm{pH} 2.5$. Low $\mathrm{pH}$ is necessary to prevent the oxidation of the $\mathrm{Fe}^{2+}$ ions to $\mathrm{Fe}^{3+}$ ions in the aqueous phase prior to the precipitation. The amount of Microemulsion II was adjusted to yield $\mathrm{pH}=11.8$ after the precipitation. A constant temperature was maintained using a water bath. The mixture of the microemulsions was aged for $1 \mathrm{~h}$ with continuous intensive stirring in ambient air, in order to let the oxidation of $\mathrm{Fe}^{2+}$ and the formation of the spinel nanoparticles. Finally, the product was washed with absolute ethanol and dried at $70{ }^{\circ} \mathrm{C}$.

The size of the nanoparticles was controlled with the amount of the surfactant $w$ and the synthesis temperature at the constant hexanol content of $55.7 \mathrm{wt} \%$ (Table 1). Samples A50, B50, and C50 were prepared at the constant temperature of $50{ }^{\circ} \mathrm{C}$, varying the parameter $w$. To synthesize the smallest nanoparticles $\mathrm{A} 50^{\prime}$, in addition to low $w$, a concentration of the reactants was decreased. The temperature during the synthesis also has a strong influence on the size and especially on the crystallinity of the nanoparticles (Košak et al. 2005). The samples B25, B50, and B95 were prepared at room temperature, $50{ }^{\circ} \mathrm{C}$, and $95{ }^{\circ} \mathrm{C}$, respectively. The largest nanoparticles B500 were prepared by annealing the as-synthesized nanoparticles B50 for $1 \mathrm{~h}$ at $500{ }^{\circ} \mathrm{C}$ in a nitrogen atmosphere. The experimental parameters of the samples are listed in Table 1.

Preparation of the standard

For comparison, the standard MnZn-ferrite spinel ceramics $\mathrm{S}$ was prepared using the classic ceramic processing (Drofenik et al. 1998). $\mathrm{Fe}_{2} \mathrm{O}_{3}, \mathrm{ZnO}$, and $\mathrm{Mn}_{3} \mathrm{O}_{4}$ powders were mixed in the ratio corresponding to the $\mathrm{Mn}_{0.5} \mathrm{Zn}_{0.5} \mathrm{Fe}_{2} \mathrm{O}_{4}$ composition, calcined for $2 \mathrm{~h}$ at $1,100{ }^{\circ} \mathrm{C}$ in air, milled, granulated, pressed into disks, and sintered for $6 \mathrm{~h}$ at $1,360{ }^{\circ} \mathrm{C}$. After sintering in air, the samples were cooled under equilibrium conditions. The X-ray powder diffractometry (XRD) of the standard showed pure spinel ferrite, and the composition of the single-phase ferrite ceramic measured with energy-dispersive X-ray spectroscopy (EDX) in a scanning electron microscope (SEM/ EDS) agreed well with the nominal composition.

\section{Characterization of nanoparticles}

The phase purity of the samples was checked using XRD (Model D4 Endeavor, Bruker AXS, Karlsruhe, Germany). The particle size, crystallinity, and composition of the nanoparticles were characterized by high-resolution electron microscopy (HREM) coupled with EDX. A field-emission electron-source transmission electron microscope TEM (JEOL 2010 F) coupled with EDS microanalysis system (LINK ISIS EDS 300) was operated at $200 \mathrm{kV}$. For the HREM investigations, the agglomerated nanoparticles were deposited on a copper-grid-supported perforated transparent carbon foil. The HREM images were taken at the edges of the nanoparticle agglomerates jutted into a hole in the carbon foil; when nanoparticles were dispersed and deposited on the carbon foil, clear HREM images could not be obtained due to their 
Table 1 Experimental conditions used during synthesis of the nanoparticles and their properties

\begin{tabular}{|c|c|c|c|c|c|c|}
\hline \multirow[t]{2}{*}{ Code } & \multirow{2}{*}{$\begin{array}{l}\text { Microemulsions } \\
\text { composition } \\
\text { Water/CTAB } \\
\text { (molar ratio) }\end{array}$} & \multicolumn{2}{|c|}{ Composition of the aqueous phases } & \multirow{2}{*}{$\begin{array}{l}\text { Temperature } \\
\left({ }^{\circ} \mathrm{C}\right)\end{array}$} & \multicolumn{2}{|c|}{ Properties of the nanoparticles } \\
\hline & & $\begin{array}{l}\text { Metal ions }{ }^{\mathrm{a}} \\
(\mathrm{mol} / \mathrm{l})\end{array}$ & $\begin{array}{l}\text { TMAH } \\
(\mathrm{mol} / \mathrm{l})\end{array}$ & & $d_{\mathrm{XRD}}(\mathrm{nm})^{\mathrm{b}}$ & $\begin{array}{l}M\left(25{ }^{\circ} \mathrm{C}\right) \\
(\mathrm{emu} / \mathrm{g})^{\mathrm{c}}\end{array}$ \\
\hline A50 & 4.46 & 0.36 & 0.5 & 50 & 1.8 & 2.4 \\
\hline $\mathrm{A} 50^{\prime}$ & 4.46 & 0.9 & 0.125 & 50 & 1.7 & 2.3 \\
\hline B25 & 9.65 & 0.36 & 0.5 & 25 & 2.2 & 2.9 \\
\hline B50 & 9.65 & 0.36 & 0.5 & 50 & 3.5 & 4.2 \\
\hline B95 & 9.65 & 0.36 & 0.5 & 95 & 8.0 & 24.7 \\
\hline $\mathrm{C} 50$ & 13.85 & 0.36 & 0.5 & 50 & 4.5 & 7.3 \\
\hline $\mathrm{B} 500^{\mathrm{d}}$ & l & / & / & 500 & 14.6 & 52.5 \\
\hline
\end{tabular}

a Total concentration of metal ions $\left(\mathrm{Zn}^{2+}+\mathrm{Mn}^{2+}+\mathrm{Fe}^{2+}\right)$

b Sizes estimated from the XRD patterns

c Specific magnetization measured at $10 \mathrm{kOe}$

d Nanoparticles B50 annealed at $500{ }^{\circ} \mathrm{C}$ in nitrogen

small size and the interference with the amorphous carbon foil beneath. In addition to the direct observation by the TEM, the particle size of the product was estimated from the X-ray diffractograms using the Debye-Scherrer method (Cullity 1987) with the crystallographic program Topas2R 2000 (Bruker AXS).

The specific magnetization of the samples was measured using a Lake Shore 7312 vibrating-sample magnetometer.

The local structure in the neighborhood of the constituent metal cations in the nanoparticles was studied by X-ray absorption spectroscopy. The MnZnferrite nanoparticles together with ceramic standard S and some oxide standards were prepared for EXAFS analysis in the form of multiple-layer powder samples on adhesive tape. EXAFS spectra at the $K$ edges of the three metallic elements were measured at the E4 beamline of HASYLAB. The E4 station provided a focused beam from an Au-coated torroidal mirror. A Si (111) double crystal monochromator with resolution of about $1 \mathrm{eV}$ was used. A dynamic higher-harmonic rejection with the feedback control of the monochromator crystals was supported by the use of a plane Au-coated mirror. The intensity of the monochromatic X-ray beam was measured by three consecutive ionization detectors of which the first was filled with nitrogen at 900 mbar and the other two with argon at a pressure of 300 mbar. The measured samples were placed between the first two detectors. The exact energy calibration with a precision of
$0.1 \mathrm{eV}$ or better was established with a simultaneous absorption measurement on the corresponding (Fe, $\mathrm{Zn}$, or $\mathrm{Mn}$ ) metal foil placed between the second and the third ionization detector.

The $K$-edge profiles of the constituent metals were scanned with a step of $0.25 \mathrm{eV}$ to enable a precise determination of the edge shape and position. For Fe and $\mathrm{Zn}$, the edge shifts were constant within experimental error. A distinct variation of the edge shift for Mn called for X-ray absorption near edge structure (XANES) measurement of a number of auxiliary samples of Mn compounds with a well-defined Mn valence.

The EXAFS spectra of the metals in the samples and the standard were measured with a conventional step progression over the interval of $1,000 \mathrm{eV}$, except for $\mathrm{Mn}\left(E_{\mathrm{K}}=6,539 \mathrm{eV}\right)$, where the proximity of the $\mathrm{Fe}$ edge $(7,112 \mathrm{eV})$ truncated the EXAFS signal at the wavevector $k$ value of $11.5 \AA^{-1}$. Similarly, due to the relatively low signal-to-noise ratio, $\mathrm{Zn}$ EXAFS spectra $\left(E_{\mathrm{K}}=9,659 \mathrm{eV}\right)$ were mostly used only to the $k$ value of $10 \AA^{-1}$. The Fe EXAFS spectra, on the other hand, were of high quality within the entire measured interval.

The spectra were analyzed with the IFEFFIT code (Ravel and Newville 2005). The FEFF model of the EXAFS signal at the three metal $K$ edges was built from the crystallographic data on the mixed ferrite (Koenig and Chol 1968). Following the argumentation in the study of Calvin et al. (2002b), a comprehensive FEFF calculation was adopted with a simultaneous 
relaxation of the three spectra, exploiting numerous connections and restraints between the parameters of the EXAFS models of the constituent elements. The advantage of this approach, with regard to the separate fitting of individual EXAFS signals, lies in the larger phase space containing roughly three times more independent data points.

\section{Results}

The diffraction patterns of the synthesized nanoparticles (Fig. 1) only consist of very broad peaks corresponding to the spinel structure. The particle sizes obtained from XRD-peak broadening are listed in Table 1. These values, although known to overshoot the average size systematically (Calvin et al. 2005), will be used throughout this article for characterization of nanoparticles. Another lower estimate will be obtained below from EXAFS data.

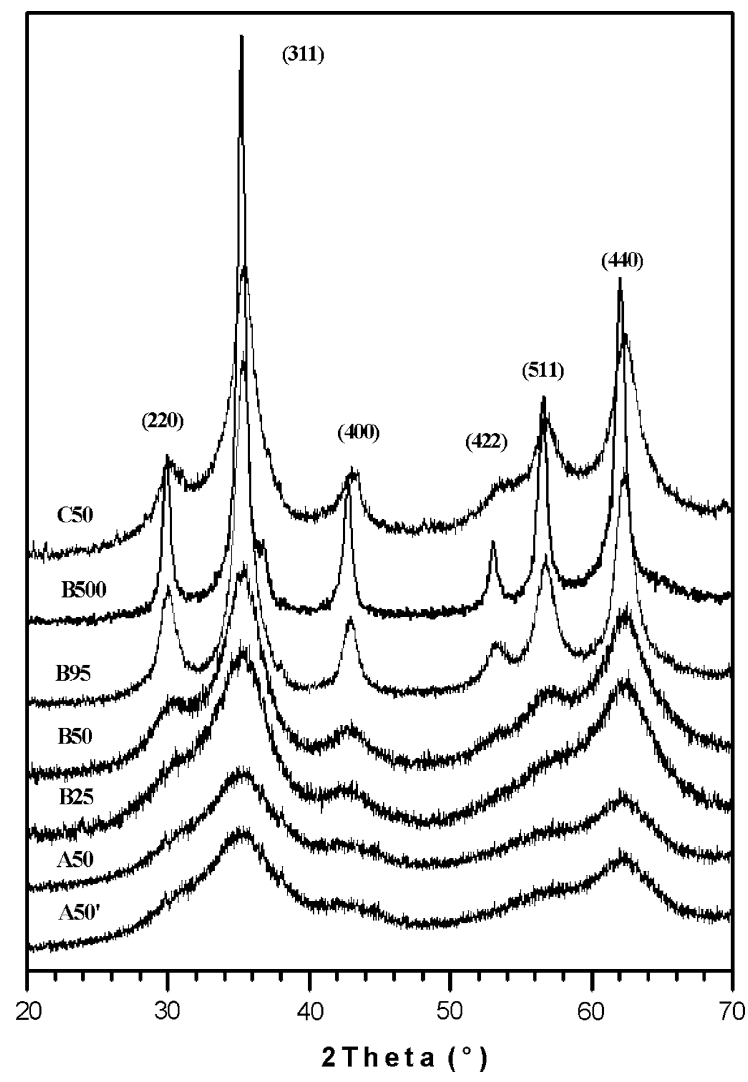

Fig. 1 Diffraction patterns of the $\mathrm{Mn}_{0.5} \mathrm{Zn}_{0.5} \mathrm{Fe}_{2} \mathrm{O}_{4}$ nanoparticles
The nanoparticle sizes obtained from XRD agree well with the sizes obtained from TEM analysis. Figure 2 shows HREM images of the smallest and the largest as-synthesized nanoparticles. The individual nanoparticles of the sample B95 $\left(d_{\mathrm{XRD}}=\right.$ $8.0 \mathrm{~nm}$ ), with appropriate orientations resolved at the edge of the agglomerate, display regular periodicity in HREM image (Fig. 2b) proving their full crystallinity. Bearing in mind the size of spinel unit cell of $\sim 0.85 \mathrm{~nm}$, it is difficult to talk about crystallinity of the smallest nanoparticles $\mathrm{A} 50^{\prime}$ $\left(d_{\mathrm{XRD}} \sim 1.7 \mathrm{~nm}\right)$, comprising just a few unit cells. The edge of the nanoparticle agglomerate appears in the HREM image almost amorphous. Some areas (marked with circles in Fig. 2a), however, exhibit tiny islands of periodicity corresponding to individual nanoparticles.

EDS analysis was used to verify the composition of the nanoparticles. The analysis of the individual nanoparticles always showed presence of all three
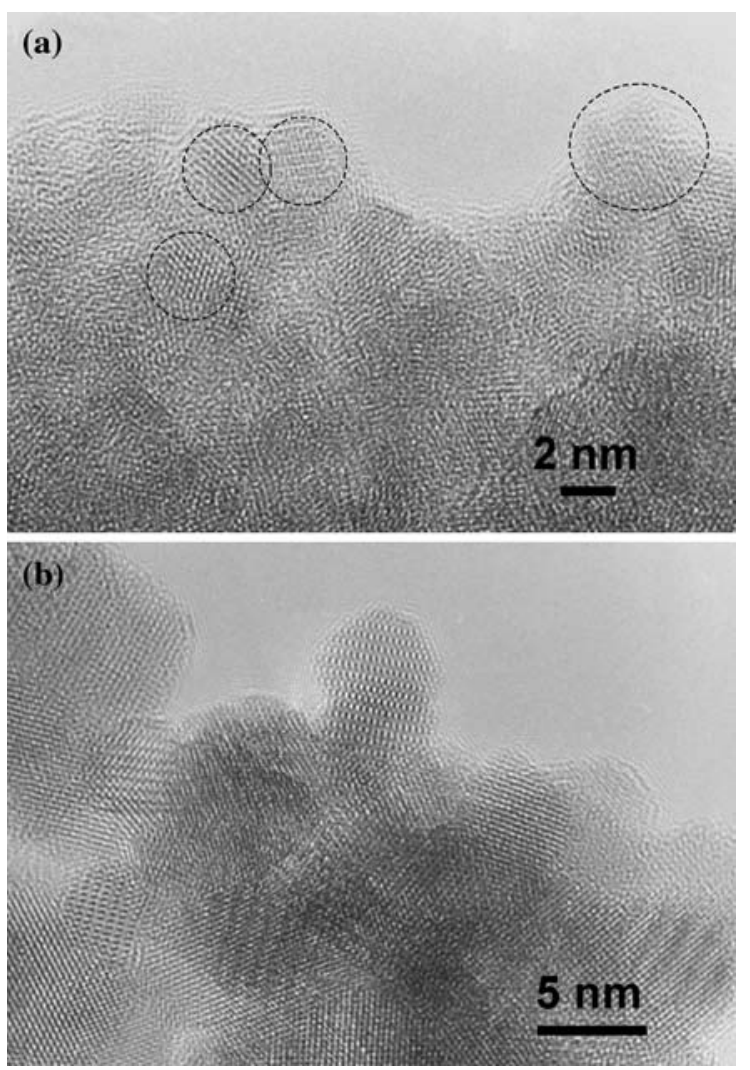

Fig. 2 HREM image of the $\mathrm{Mn}_{0.5} \mathrm{Zn}_{0.5} \mathrm{Fe}_{2} \mathrm{O}_{4}$ nanoparticles A50' (a) and B95 (b) 
constituting cations in the ratio that reflected approximately the starting $\mathrm{Mn}_{0.5} \mathrm{Zn}_{0.5} \mathrm{Fe}_{2} \mathrm{O}_{4}$ composition.

The room-temperature measurements of specific magnetization as a function of magnetic field $(M-H)$ (Fig. 3) show ferrimagnetism for larger nanoparticles B50, B95, C50, and B500. Their magnetization hystereses show zero coercitivity suggesting a superparamagnetic nature. Even at relatively high fields, the saturation magnetization is not reached, in agreement with the existence of a magnetically inactive layer at the surface (Kodama 1999). The proportion of this non-magnetic contribution increases with decreasing particle size, and as a result, the magnetization measured at a high field (Table 1) decreases in parallel with the decrease in the size. For the nanoparticle size below approximately $3 \mathrm{~nm}$ (samples $\mathrm{A} 50, \mathrm{~A} 50^{\prime}$, and $\mathrm{B} 25$ ), the magnetically inactive surface layer completely prevails and the sample is paramagnetic (see inset of Fig. 3).

XANES analysis shows pure Fe(III) and Zn(II) valence in all measured samples and a considerable variation in Mn $K$-edge shifts. Figure 4 shows $\mathrm{Mn}$ and Fe XANES profiles for the synthesized nanoparticles and some standard compounds. From the spectra of the reference samples with known manganese oxidation states, we have found that the $\mathrm{Mn}$ $K$ edge is shifted for about $3.5 \mathrm{eV} /$ valence unit in agreement with previous observations (Zabukovec Logar et al. 2006).

The average valence of $\mathrm{Mn}$ in the $\mathrm{MnZn}$-ferrite ceramic standard $\mathrm{S}$ was determined from $\mathrm{Mn} K$-edge

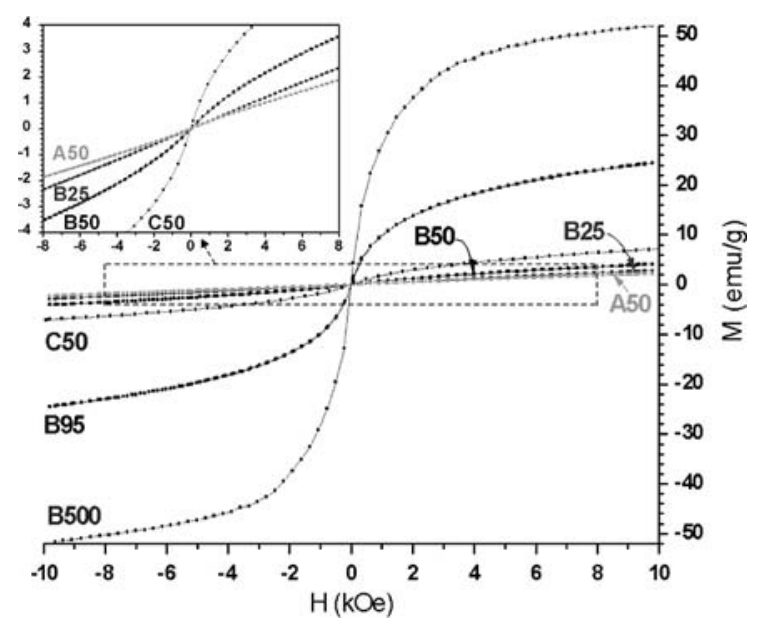

Fig. 3 Room-temperature magnetization hysteresis of the $\mathrm{Mn}_{0.5} \mathrm{Zn}_{0.5} \mathrm{Fe}_{2} \mathrm{O}_{4}$ nanoparticles position to be $2.7+$, similar to the valence of $\mathrm{Mn}$ in manganese oxide hausmanite $\left(\mathrm{Mn}_{3} \mathrm{O}_{4}\right)$, which also has a spinel-related structure. The incorporation of the manganese in a ferrite spinel in a mixture of $\mathrm{Mn}(\mathrm{II})$ and $\mathrm{Mn}$ (III) states is not unusual. The same valence is found in the annealed nanoparticles B500.

In the as-synthesized nanoparticles, the Mn $K$ edge is shifted toward higher energies, indicating an increase in the average valence (Table 2). The value $3.0+$ is found in the largest as-synthesized nanoparticles B95, whereas in all other smaller nanoparticles it exceeds this value, indicating the presence of some $\mathrm{Mn}(\mathrm{IV})$. The shape of the absorption edge also indicates that $\mathrm{Mn}$ valence higher than $3+$ measured in the nanoparticles is a consequence of the combination of $\mathrm{Mn}(\mathrm{III})$ and $\mathrm{Mn}(\mathrm{IV})$.

The local structure of the nanoparticles has been studied using EXAFS and compared to the structure of the ceramic standard. Some conclusions on the structure of the nanoparticles can already be made by inspection of the Fourier transforms of the EXAFS spectra (Fig. 5). The positions of the Fourier (FT) peaks, after a small phase correction, correspond to the bond distance between the absorbing and the backscattering atom. The first peak in the FT EXAFS spectra around $\sim 1.6 \AA$ corresponds to first neighbors-oxygen ions comprising the oxygen polyhedra. (The corresponding interatomic distance after phase correction is close to $2 \AA$-the same for all three metal ions.) The scattering from the second neighbors forms the next wide peak in the FT EXAFS spectra, apparently composed of two components or subpeaks centered at $\sim 2.6$ and $\sim 3.1 \AA$ (corresponding to real distances of 3.0 and $3.6 \AA$, respectively). The relative amplitudes of those subpeaks depend strongly on the site of the absorber cation. The octahedral site has six second neighbors at the distance of $3.0 \AA$ and six at the distance of $3.5 \AA$. The cation at a tetrahedral site has 12 second neighbors at the distance of $3.5 \AA$ and four second neighbors at the distance of $3.6 \AA$. Thus, a subpeak in the FT EXAFS centered at $\sim 2.6 \AA$ (marked in Fig. 5 with B) indicates the incorporation of the absorber cation at the octahedral or B-site, while the subpeak centered at $3.1 \AA$ (marked in Fig. 5 with $\mathrm{A}$ ) indicates the absorber cation incorporated at a tetrahedral lattice site (A-site). By comparing the intensities of the A and B peaks in Fig. 5, it could be concluded that in the ceramic standard $\mathrm{S}, \mathrm{Zn}$ is incorporated preferentially at tetrahedral A-sites, 
Fig. $4 \mathrm{Mn}$ (a) and $\mathrm{Fe}(\mathbf{b})$ XANES spectra measured on $\mathrm{Mn}_{0.5} \mathrm{Zn}_{0.5} \mathrm{Fe}_{2} \mathrm{O}_{4}$ ceramic standard $(\mathrm{S})$, on synthesized nanoparticles and on references: $\mathrm{Mn}$ metal, $\mathrm{MnO}, \mathrm{Mn}_{3} \mathrm{O}_{4}, \mathrm{MnO}_{2}$, $\mathrm{K}_{3}\left[\mathrm{Mn}^{3+}\left(\mathrm{C}_{2} \mathrm{O}_{4}\right)_{3}\right] \cdot 3 \mathrm{H}_{2} \mathrm{O}$ (a standard for $\mathrm{Mn}(\mathrm{III})$ ), $\mathrm{Fe}_{2} \mathrm{O}_{3}$, and $\mathrm{LiFePO}_{4}$ (a standard for $\mathrm{Fe}(\mathrm{II})$ )
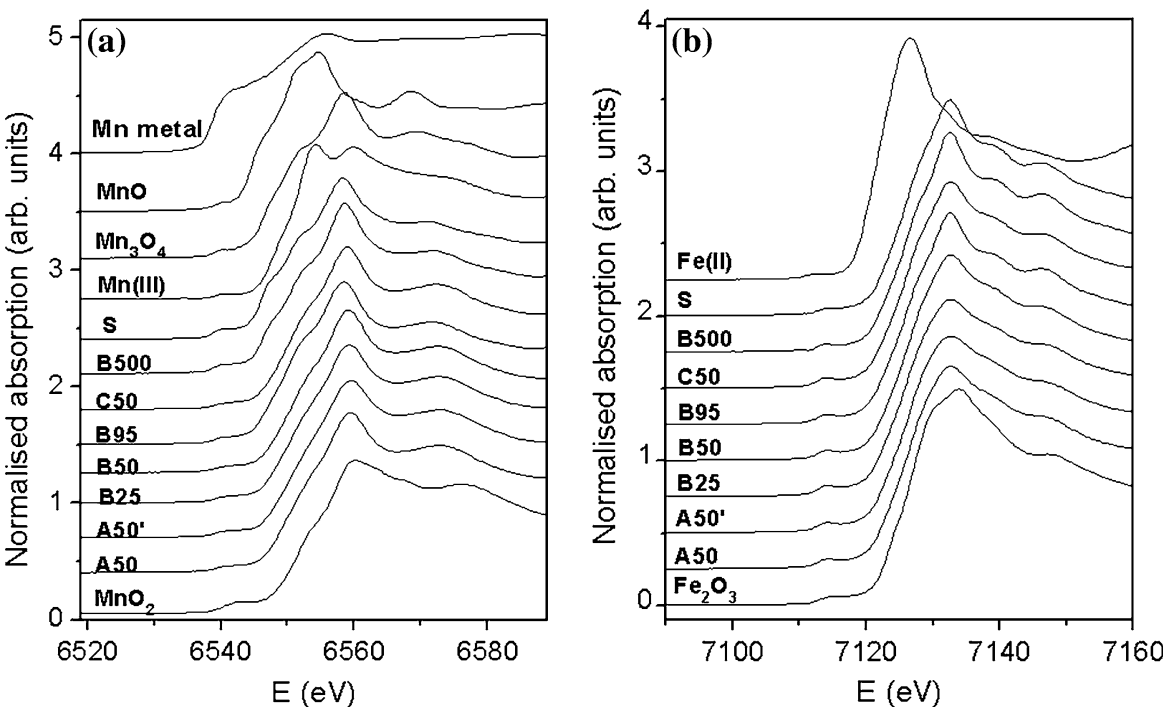

Table 2 Average valence of $\mathrm{Mn}$ in the nanoparticles extracted from XANES and the least-square values of EXAFS parameters: the occupation of $\mathrm{Mn}$ and $\mathrm{Zn}$ at the octahedral B-sites $\left(\mathrm{Mn}_{\mathrm{B}}\right.$ and $\left.\mathrm{Zn}_{\mathrm{B}}\right)$, the average second neighbor yields $q$, the derived nanoparticle diameter $d_{\mathrm{XAFS}}$, and the quality of fit ( $r$-factor)

\begin{tabular}{lllllllll}
\hline Sample & A50 & A50' & B25 & B50 & B95 & C50 & B500 & S \\
\hline$d_{\text {XRD }}(\mathrm{nm})$ & 1.8 & 1.7 & 2.2 & 3.5 & 8.0 & 4.5 & 14.6 & Bulk \\
Mn valence & $3.3+$ & $3.3+$ & $3.3+$ & $3.3+$ & $3.0+$ & $3.2+$ & $2.7+$ & $2.7+$ \\
Mn $_{\text {B }}$ & $1.0(6)$ & 1.0 & 1.0 & 0.92 & 0.96 & $\sim 1$ & 0.69 \\
$\mathrm{Zn}_{\mathrm{B}}$ & $0.3(1)$ & 0.0 & 0.3 & 0.0 & 0.0 & 0.2 & 0.0 & 0.45 \\
$q$ & $0.58(7)$ & 0.65 & 0.58 & 0.72 & 0.81 & 0.82 & 1 & 1 \\
$d_{\text {XAFS }}(\mathrm{nm})$ & $1.1(2)$ & 1.3 & 1.1 & 1.6 & 2.4 & 2.5 & $\infty$ & $\infty$ \\
$r$-Factor & 0.050 & 0.018 & 0.053 & 0.024 & 0.056 & 0.060 & 0.032 \\
\hline
\end{tabular}

The typical error of the last digit is given in parentheses in the first column

while $\mathrm{Fe}$ is situated preferentially at octahedral B-sites. Mn is incorporated at both lattice sites.

In the same qualitative vein, the $\mathrm{Fe}, \mathrm{Zn}$, and $\mathrm{Mn}$ FT EXAFS spectra of the standard $\mathrm{S}$ and the different nanoparticle samples are compared in Fig. 6a-f. The data are grouped into two series, each displaying the effect on the nanoparticle size of a single parameter in the synthesis route: the surfactant molar ratio (A50, $\left.\mathrm{A} 50^{\prime}, \mathrm{B} 50, \mathrm{C} 50\right)$ and the temperature (B25, B50, B95, B500). The quality of the experimental material can be judged from the $k$-space EXAFS signal, the $k^{3} \chi(k)$ graphs given in the supplement.

The positions of the first peaks in the spectra remain almost unchanged with the particle size of the samples. They belong to the oxygen neighbors at respective contact distances, which do not change appreciably with the size of the particles. The amplitude of the first peaks, showing the number of first neighbors, however, can depend on the particle size: very slightly for the Fe atoms, somewhat more for the $\mathrm{Zn}$ atoms, and strongly for the Mn atoms. Since metal ions get fully dressed by the oxygen atoms, the changes are best explained by the transition of the ions between the sites, as will be confirmed later in the quantitative analysis. The stoichiometry $\mathrm{Mn}: \mathrm{Zn}: \mathrm{Fe}=1: 1: 4$ shows that Fe ions remain dominantly on $\mathrm{B}$-sites with six $\mathrm{O}$ neighbors. $\mathrm{Zn}$ atoms switch to B-sites infrequently and $\mathrm{Mn}$ atoms quite often.

The amplitudes of the second peaks at the larger distances decrease drastically with the decreasing particle size. This can be interpreted as a loss of second neighbors, either due to the incomplete neighborhood of the (near) surface atoms or due to 


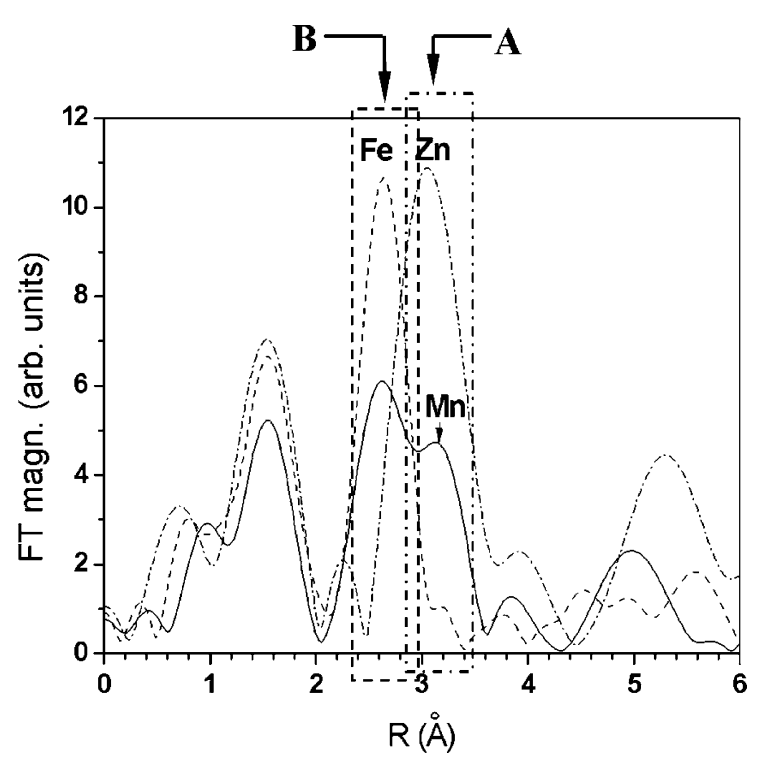

Fig. 5 FT EXAFS $k^{3}$-weighted spectra from Fe, Zn, and Mn $K$ edge in the MnZn-ferrite ceramic standard S

the loss of coherence of the EXAFS signal induced by the change of bond lengths at the surface.

Crucial for understanding of the nanoparticle structure is the two second-neighbor subpeaks. The two narrow vertical rectangles $\mathrm{A}$ and $\mathrm{B}$ show the ranges of distances to the octahedral and tetrahedral sites, respectively, largely independent on the site of the central atom.

In $\mathrm{Zn}$ spectra the second neighbors form one peak, centered at $\sim 3.1 \AA$ (marked in Fig. 6 with A), suggesting that $\mathrm{Zn}$ atoms remain mostly on A-sites. In $\mathrm{Fe}$ spectra, a significant relative increase in the amplitude of the A-peak combined with a simultaneous relative decrease of the amplitude of the B-peak can be noted as the particle size decreases, suggesting that an increasing proportion of Fe atoms changes sites from octahedral B to tetrahedral A positions. In Mn spectra, the A-peak is very small in the case of the larger as-synthesized nanoparticles B95 and C50, whereas it is missing completely for the smaller nanoparticles A50, A50', B25. Obviously, the $\mathrm{Mn}$ atoms are incorporated into the structure of the nanoparticles predominantly at octahedral B-sites.

The FT EXAFS of the nanoparticles B500 annealed at $500{ }^{\circ} \mathrm{C}$ are similar to the spectra of the ceramic standard suggesting only minor differences in the structure of the both materials.
Fig. 6 FT EXAFS $k^{3}$-weighted spectra ( $k$ of Fe, Zn, and Mn $K$ edge in the MnZn-ferrite nanoparticles: the spectra of the nanoparticles of different size synthesized at a constant temperature of $50{ }^{\circ} \mathrm{C}$ are compared in (a), (c), and (e), whereas the spectra of the nanoparticles prepared at different temperatures are compared in (b), (d), and (f). The spectra of three metal ions are displaced vertically so that the distances to the neighbors can be directly compared

To give these observations a firm quantitative basis, the EXAFS spectra are modeled with an ab initio FEFF model (Ravel and Newville 2005). Sufficient flexibility of the model is required to account for the deformation of the structure in the small nanoparticles with a large proportion of surface atoms with incomplete neighborhood. Additional degrees of freedom are needed in the model to describe swapping of the three metal ions between the octahedral (B) and tetrahedral (A) sites, with subsequent adaptation of the unit cell. Thus, the model introduces adaptable occupation probabilities of the two sites by the metal ions and adaptable inter-site distances in addition to the standard set of EXAFS model parameters for each metal ion: coordination numbers, neighbor distances, and Debye-Waller widths of individual scattering paths. With all these, the model is not solvable in the routine manner, i.e., with separate least-square fits of the model parameters to the three metal spectra. Even if satisfactory individual fits are obtained, the values of the shared parameters, as a rule, will not be consistent between the three sets. For example, the scattering path $\mathrm{Fe}-\mathrm{Mn}$ contributes to Fe as well as Mn spectra, and the same length and Debye-Waller width, with related amplitudes, should be obtained. A systematic way to honor such constraints is a simultaneous fit of three corresponding spectra. Calvin et al. (2002b) have demonstrated it successfully in a similar study of MnZn-ferrite spinel structure in dependence of preparation route. They have built a minimum structure model whereby positions of atoms are calculated from the crystallographic data, expressed with just two parameters: the overall expansion coefficient and the oxygen parameter. The latter, with the range between 0.375 and 0.39 , only affects the positions of oxygen atoms in the unit cell. In addition to the intrinsic EXAFS parameters, the energy shifts, and the amplitude reduction factors for the three metal ions, they have also adopted three Debye-Waller widths for the metal-oxygen bonds and another one for all intermetal distances. The metal-site occupation probabilities are 

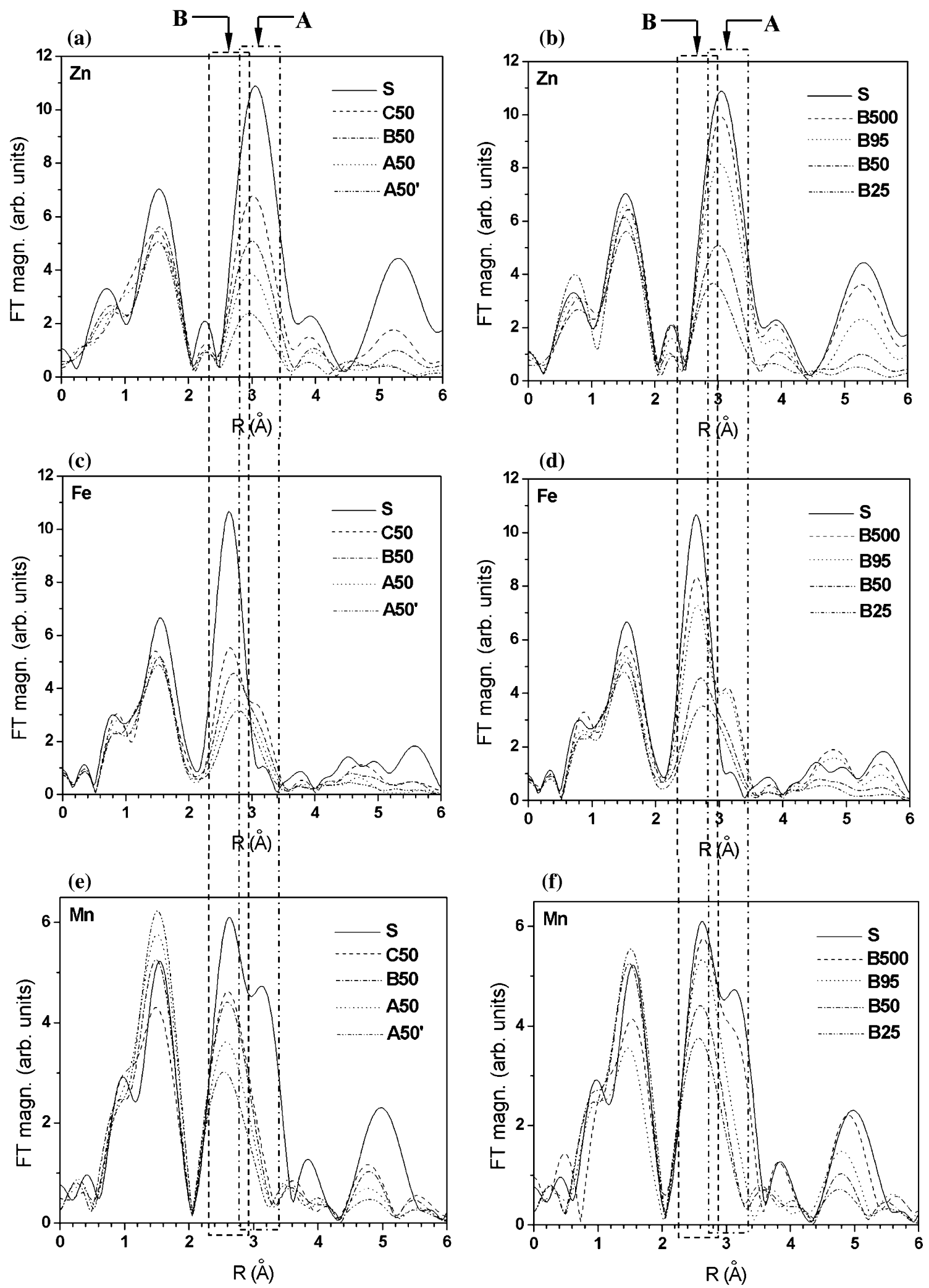
all connected and can be expressed by just two of them. Similarly, only two corrections to the tabulated metaloxygen distances are required, making 16 parameters in all. The authors also list the assumptions in the model: no impurities and no vacancies in the crystallographic structure, random occupation of sites within given probabilities, no correlation between valence and occupation.

For our purpose, the model has to be expanded to accommodate the particle-size dependence. It turned out in preliminary investigations that some additional information can be extracted: so we dispensed with the limitation of a unique Debye-Waller width for all neighbors beyond the first and introduced separate widths for $\mathrm{B}-\mathrm{B}, \mathrm{B}-\mathrm{A}$, and $\mathrm{A}-\mathrm{A}$ paths.

For the adapting inter-metal distances, we introduced a set of $\mathrm{B}-\mathrm{B}, \mathrm{B}-\mathrm{A}$, and $\mathrm{A}-\mathrm{A}$ distance corrections, restrained to a zero average, in order not to spoil the meaning of the overall expansion parameter. Another novelty are the yields of the second neighbors, separate for $\mathrm{B}-\mathrm{B}, \mathrm{B}-\mathrm{A}$, and $\mathrm{A}-\mathrm{A}$ neighbor pairs, to describe the incomplete neighborhood in small particles.

With all the cloning of photoelectron scattering paths required to describe all possible site occupations and neighbor relations, $2 \times 10$ FEFF paths are necessary to model the EXAFS signal of each element in the range of first and second neighbors, i.e., within $3.7 \AA$. This range includes two multiplescattering paths in each decade, both negligible after the inspection of the compound model EXAFS signal and its contributions.

Figures 7 and 8 show the results of least-square relaxation of the EXAFS spectra of the standard S and the worst case, the smallest nanoparticles $\mathrm{A} 50^{\prime}$, to the model. The complete set of EXAFS parameters is given in the supplement table for the $k^{3}$ weight and $k$-ranges (in $\AA^{-1}$ ) of 3.7-10.5 (Mn), 3.7-12.5 (Fe), and $3.5-9.7(\mathrm{Zn})$, and $R$-ranges (in $\AA$ ) of $1.1-3.5$ $(\mathrm{Mn}), 1.1-3.6(\mathrm{Fe})$, and 1.1-3.7 (Mn). The values of crucial structural parameters of all samples are resumed in Table 2. The quality of the fits ( $r$-factor) is satisfactory, mostly in the range of $5 \%$. This is however only the overall figure: the Fe spectra as a rule give much better fits, below $1 \%$, while the agreement of the $\mathrm{Zn}$ data is always the poorest, mostly close to $10 \%$. Much better fits of Fe spectra compared to those of $\mathrm{Zn}$ and $\mathrm{Mn}$, especially for spectra collected at smaller nanoparticles, can be
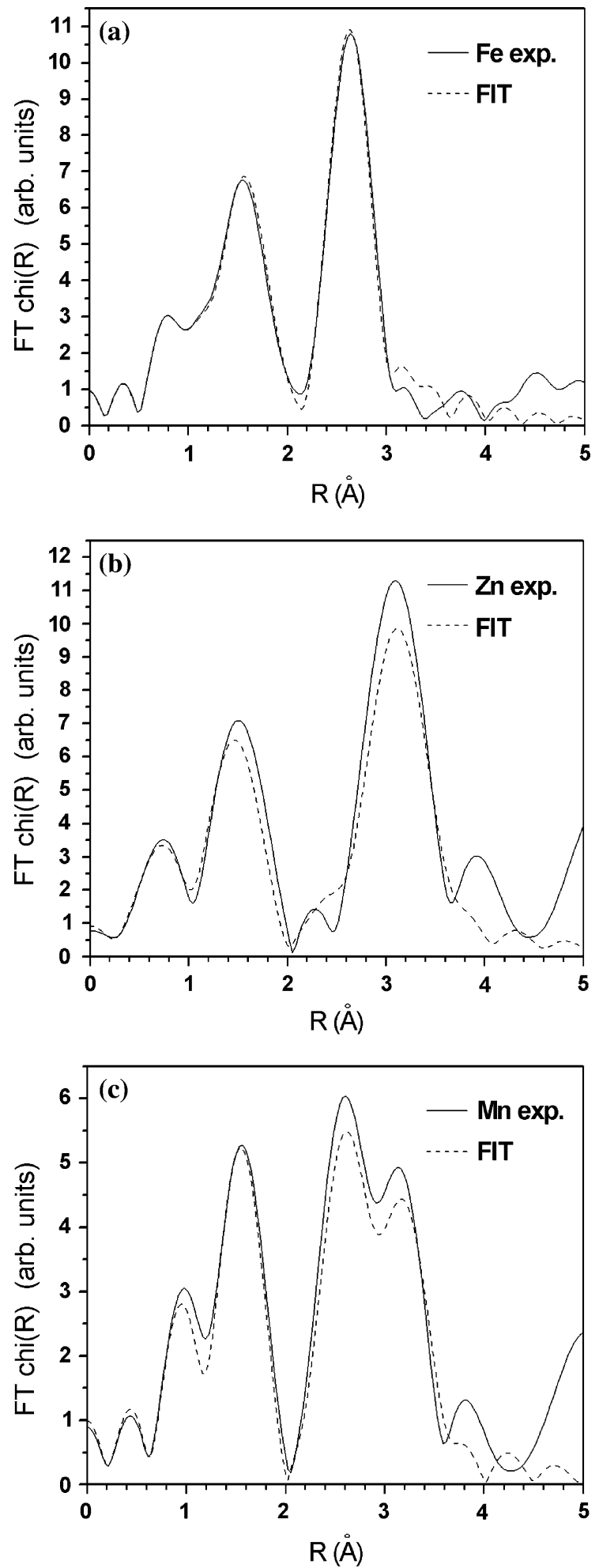

Fig. 7 FT magnitude of the $k^{3}$-weighted Fe (a), Zn (b), and Mn (c) EXAFS data (solid line) with the model (dashed) for the standard $\mathrm{S}$ 

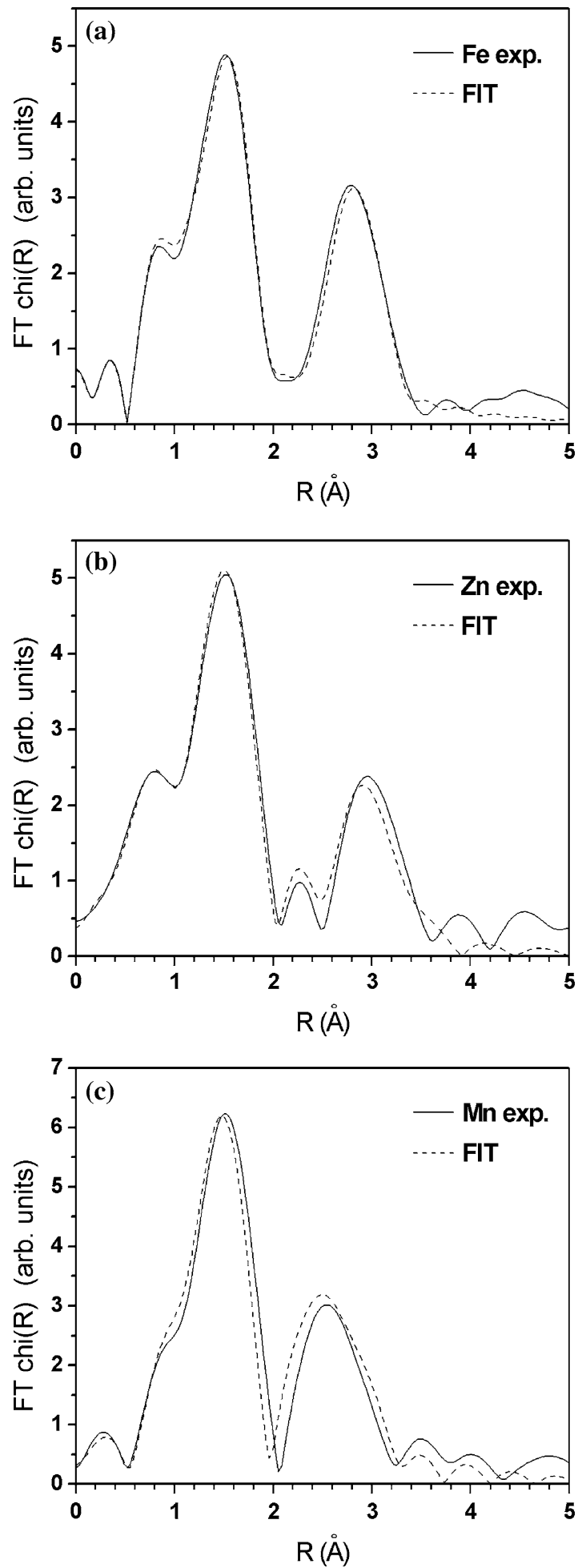

Fig. 8 FT magnitude of the $k^{3}$-weighted Fe (a), Zn (b), and Mn (c) EXAFS data (solid line) with the model (dashed) of the smallest nanoparticles $\mathrm{A}^{\prime} 0^{\prime}\left(d_{\mathrm{XRD}}=1.7 \mathrm{~nm}\right)$ related to the better quality of the Fe data. However, they may also suggest that $\mathrm{Fe}$ atoms are situated at the positions close to those of the "ideal" spinel structure, while the positions of the other two cations deviate more. The differences in the quality of the fits for different metal ions therefore suggest a structure based on more rigid lattice occupied by $\mathrm{Fe}$ ions at positions typical for the ideal spinel structure, in which $\mathrm{Mn}$ and $\mathrm{Zn}$ atoms are more loosely incorporated.

Many of the intrinsic EXAFS parameters, given only in the supplement table, e.g., the energy shifts of the ions and the Debye-Waller factors of the scattering paths, exhibit small, apparently random variations around some average value, typical of all samples. Steady trends with the estimated size $d_{\text {XRD }}$ can be established for the newly introduced parameters describing the site swapping and the loss of neighbors. The probability of $\mathrm{Mn}$ atom occupying the $\mathrm{B}$-site, $\mathrm{Mn}_{\mathrm{B}}$, correlates clearly with the estimated size of the particles. From the bulk value of 0.45 , it raises to 0.69 in the large B500 particles. In smaller particles the exchange is almost complete; the values just below 1 can be discerned in B50 and B95 samples. The yields of second neighbors qoo, qot, and qtt, accessible in the supplement, show considerable variation among site pairs $\mathrm{AA}, \mathrm{AB}$, and $\mathrm{BB}$, but their mean $q$, given in Table 2, correlates directly with the particle size, changing from 0.58 for the smallest particles to the expected 1.0 in the bulk.

The site-swapping parameter for $\mathrm{Zn}, \mathrm{Zn}_{\mathrm{B}}$, gives less definite results. It is the least robust parameter of the model, strongly coupled to other parameters and unstable in the relaxation procedure, prone to escaping out of the physically sensible range. The values in the table are mostly obtained by restraining the fits and should be regarded with some reserve. It is entirely possible that the proper values should all be close to zero (no site exchange).

\section{Discussion}

The spinel structure of the small $\mathrm{Mn}_{0.5} \mathrm{Zn}_{0.5} \mathrm{Fe}_{2} \mathrm{O}_{4}$ nanoparticles synthesized with the co-precipitation in microemulsions was found to differ distinctly from that of the bulk ceramic standard (Table 2). According to the EXAFS results, the structure of the ceramic 
standard can be expressed with the $\left(\mathrm{Zn}_{0.50} \mathrm{Mn}_{0.27} \mathrm{Fe}_{0.23}\right)\left[\mathrm{Fe}_{1.77} \mathrm{Mn}_{0.23}\right] \mathrm{O}_{4}$ formula (round and square brackets represent tetrahedral and octahedral sites, respectively), displaying the degree of inversion $X=0.23$. The average $\mathrm{Mn}$ valence was determined as 2.7 by XANES analysis, suggesting a combination of $\mathrm{Mn}$ (II) and $\mathrm{Mn}$ (III). It is expected that the $\mathrm{Mn}(\mathrm{II})$ ions preferentially occupy the larger octahedrally coordinated interstitials and the Mn(III) ions the smaller tetrahedrally coordinated interstitials.

EXAFS shows a minor change from the bulk structure, when the material is prepared in the form of larger nanoparticles $\left(d_{\mathrm{XRD}}=14.6 \mathrm{~nm}\right)$ by annealing of the as-synthesized nanoparticles at the elevated temperature of $500{ }^{\circ} \mathrm{C}$. The migration of $\mathrm{Mn}$ from A-sites to B-sites (and migration of $\mathrm{Fe}$ in the opposite direction) is stronger, resulting in the increased degree of inversion $X=0.35$. XANES shows no change in the average Mn valence.

The changes in the structure are much larger in the as-synthesized nanoparticles. The degree of inversion increases further with the decrease in size. The migration of $\mathrm{Mn}$ from A-sites to B-sites is complete or almost complete.

Moreover, with the decreasing nanoparticle size, the average $\mathrm{Mn}$ valence increases above $3+$ to reach values of $3.3+$ in the very small nanoparticles, suggesting an unusual combination of $\mathrm{Mn}$ (III) and $\mathrm{Mn}(\mathrm{IV})$. The presence of $\mathrm{Mn}(\mathrm{IV})$ valence state incorporated in the spinel ferrite structure is extremely rare and has not been observed in bulk ferrite spinel materials. A careful analysis of the nanoparticles using TEM/EDS showed that each individual nanoparticle contains all three constituting cations $\mathrm{Fe}$, $\mathrm{Zn}$, and $\mathrm{Mn}$ in the ratio which within the accuracy of the analysis reflects the nanoparticle nominal composition $\mathrm{Mn}_{0.5} \mathrm{Zn}_{0.5} \mathrm{Fe}_{2} \mathrm{O}_{4}$. Mn-rich nanoparticles, indicating the presence of $\mathrm{MnO}_{2}$, were never detected. So, it could be concluded with confidence that $\mathrm{Mn}(\mathrm{IV})$ is incorporated into the spinel nanoparticles.

Since all metal ions in a nanoparticle presumably reside in complete oxygen polyhedra so that the outer layer of the particle is entirely covered with oxygen, there should be a stoichiometric excess of oxygen. This requires additional charge on the cations. Since $\mathrm{Fe}$ and $\mathrm{Zn}$ are already in their highest respective valence states as also evidenced by the Fe XANES in Fig. $4 \mathrm{~b}$, it is only Mn that can adapt to a higher oxidation state. In this view, the Mn(IV) state has to be regarded as a surface, rather than structural, effect of the particle size.

The second neighbor yield $q$ provides a clue to the question, whether the observed changes in the structure of the nanoparticles relate to the whole particle or merely to the surface? The X-ray absorption techniques used in the study provide the information on the neighborhood of an average atom, not resolving between the presumably better-defined core of the particle and the less well-ordered surface. In the smallest nanoparticles with the diameter of a few unit cells, a considerable proportion of the atoms sits at the surface. These surface atoms have incomplete neighborhood and a wider range of bond distances. The yield $q$ shows that the ions in the smallest nanoparticles have on average only $60 \%$ of the bulk number of the second (metal) neighbors. The number also gives an estimate to what extent can the other structure parameters be affected.

An additional insight into the question is given by the partial neighbor yields, pertaining to $\mathrm{AA}, \mathrm{AB}$, and $\mathrm{BB}$ neighbor pairs. Although the partial values exhibit much less clear trends, the $\mathrm{BB}$ yields are consistently the highest. It is an indication that the $\mathrm{B}$-sites are formed at the surface earlier than A-sites and with less deformation.

The neighbor yields $q$ can also be exploited for an estimate of the nanoparticle size. Even if the number of first neighbors is not affected by the size, the number of the second neighbors is defective in small particles. Rather than relying on asymptotic formulae appropriate for large particle sizes, the yield can be related to the particle diameter by counting the neighbors in a model particle (Kodre et al. 1999). Such estimates depend on the shape of the model particle and are generally regarded as lower-bound estimates: for our $q$ values and a spherical particle model, they are given in Table 2. The values are consistently smaller than the Debye-Scherrer estimates for a factor between 1.5 and 3; the relation between the different size estimates has been comprehensively discussed by Calvin et al. (2005).

The analysis could not detect any significant difference in the structural changes between the nanoparticles of different sizes prepared at the constant temperature of $50{ }^{\circ} \mathrm{C}$ and those prepared at different temperatures. This suggests that the nanoparticle size itself has the dominant effect on the 
structure and that the direct impact of thermodynamic conditions, such as the synthesis temperature, is much less pronounced.

\section{Conclusions}

The structure of small $\mathrm{Mn}_{0.5} \mathrm{Zn}_{0.5} \mathrm{Fe}_{2} \mathrm{O}_{4}$ spinel-ferrite nanoparticles synthesized using co-precipitation in reversed microemulsions has been studied as a function of their Scherrer size ranging from $\sim 2$ to $\sim 15 \mathrm{~nm}$ using X-ray absorption techniques of EXAFS and XANES.

When prepared in the form of nanoparticles, the structure of $\mathrm{Mn}_{0.5} \mathrm{Zn}_{0.5} \mathrm{Fe}_{2} \mathrm{O}_{4}$ ferrite acquires a different distribution of the constituting cations over two sublattices of the spinel structure. These changes are dominated by the migration of $\mathrm{Mn}$ ions from the tetrahedral A to the octahedral B-sites, compensated by a counter-migration of $\mathrm{Fe}$, and an increase in $\mathrm{Mn}$ valence above $3+$, suggesting partial oxidation of $\mathrm{Mn}$ in $\mathrm{Mn}(\mathrm{IV})$ valence state. In the nanoparticles below $8 \mathrm{~nm}, \mathrm{Mn}$ is completely incorporated at the B-sites. The migration of $\mathrm{Zn}$ is sporadic and uncertain. Annealing at $500{ }^{\circ} \mathrm{C}$ relaxes the structure of as-synthesized nanoparticles toward the structure of the ceramic bulk standard.

Acknowledgments This work was supported by the Slovenian Research Agency, the Ministry of Higher Education, Science and Technology of the Republic of Slovenia within the National Research Program and by DESY and the European Community under Contract RII3CT-2004-506008 (IA-SFS). Provision of synchrotron radiation facilities by HASYLAB (project II-04-065 EC) is acknowledged. The authors would also like to thank Dr. Aljoša Košak for help with the synthesis of the nanoparticles and E. Welter of HASYLAB for expert advice on beamline operation.

\section{References}

Ammar S, Jouini N, Fievet F, Stephan O, Marhic C, Richard M, Villain F, Chartier dit Moulin Ch, Brice S, Sainctavit Ph (2004) Influence of the synthesis parameters on the cation distribution of $\mathrm{ZnFe}_{2} \mathrm{O}_{4}$ nanoparticles obtained by forced hydrolysis in polyol medium. $\mathrm{J}$ Non-Cryst Solids 345\&346:658-662

Batlle X, Labarta A (2002) Finite-size effect in fine particles: magnetic and transport properties. J Phys D Appl Phys 35:R15-R42
Calvin S, Carpenter EE, Harris VG, Morrison SA (2002a) Use of multiple-edge refinement of extended X-ray absorption fine structure to determine site occupancy in mixed ferrite nanoparticles. Appl Phys Lett 81:3828-3830

Calvin S, Carpenter EE, Ravel B, Harris VG, Morrison SA (2002b) Multiedge refinement of extended X-ray absorption fine structure of manganese zinc ferrite nanoparticles. Phys Rev B 66:224405-1-224405-13

Calvin S, Luo SX, Caragianis-Broadbridge C, McGuinness JK, Anderson E, Lehman A, Wee KH, Morrison SA, Kurihara LK (2005) Comparison of extended X-ray absorption fine structure and Scherrer analysis of X-ray diffraction as methods for determining mean sizes of polydisperse nanoparticles. Appl Phys Lett 87:233102-1233102-3

Carpenter EE, O'Connor CJ, Harris VG (1999) Atomic structure and magnetic properties of $\mathrm{MnFe}_{2} \mathrm{O}_{4}$ nanoparticles produced by reverse micelle synthesis. J Appl Phys 85:5175-5177

Chen JP, Sorensen CM, Klabunde KJ, Hadjipanayis GC, Devlin E, Kostikas A (1996) Size-dependent magnetic properties of $\mathrm{MnFe}_{2} \mathrm{O}_{4}$ fine particles synthesized by coprecipitation. Phys Rev B 54:9288-9296

Cullity BD (1987) Elements of X-ray diffraction. AddisonWesley, Reading

Drofenik M, Žnidaršič A, Makovec D (1998) Influence of the addition of $\mathrm{Bi}_{2} \mathrm{O}_{3}$ on the grain growth and magnetic permeability of MnZn ferrites. J Am Ceram Soc 81:28412848

Fetami DJ, Harris VG, Browning VM, Kirkland JP (1998) Processing and cation redistribution of MnZn ferrites via high-energy ball milling. J Appl Phys 83:6867-6869

Hamdeh HH, Ho JC, Oliver SA, Willey RJ, Oliveri G, Busca GJ (1997) Magnetic properties of partially-inverted zinc ferrite aerogel powders. J Appl Phys 81:1851-1857

Jeyadevan B, Tohji T, Nakatsuka KJ (1994) Structure-analysis of coprecipitated $\mathrm{ZnFe}_{2} \mathrm{O}_{4}$ by extended X-ray absorption fine-structure. Appl Phys 76:6325-6327

Jeyadevan B, Tohji T, Nakatsuka K, Narayanasamy A (2000) Irregular distribution of metal ions prepared by co-precipitation technique structure analysis of $\mathrm{Mn}-\mathrm{Zn}$ ferrite using extended X-ray absorption fine structure. J Magn Magn Mater 217:99-105

Kamiyama T, Haneda K, Sato T, Ikeda S, Asano H (1992) Cation distribution in $\mathrm{ZnFe}_{2} \mathrm{O}_{4}$ fine particles studied by neutron powder diffraction. Solid State Commun 81:563-566

Kodama RH (1999) Magnetic nanoparticles. J Magn Magn Mater 200:359-372

Kodre A, Arčon I, Batista J, Pintar A (1999) EXAFS analysis of Pd atomic clusters. J Synchrotron Radiat 6:458-459

Koenig U, Chol G (1968) Roentgenbeugungs- und Neutronenbeugungsuntersuchungen an Ferriten der Reihe $\mathrm{Mn}_{\mathrm{x}} \mathrm{Zn}_{1-\mathrm{x}} \mathrm{Fe}_{2} \mathrm{O}_{4}$. J Appl Crystallogr 1:124-126

Košak A, Makovec D, Drofenik M (2005) Preparation of spinel ferrite nano-particles using precipitation in water-in-oil microemulsions. J Metastable Nanocryst Mater 23:251-254

Makovec D, Košak A, Drofenik M (2004) The preparation of MnZn-ferrite nanoparticles in water-CTAB-hexanol microemulsions. Nanotechnology 15:S160-S166

Pileni MP (1993) Reverse micelles as microreactors. J Phys Chem 97:6961-6973 
Ravel B, Newville M (2005) ATENA, ARTHEMIS, HEPHAESTUS: data analysis for X-ray absorption spectroscopy using IFEFFIT. J Synchrotron Radiat 12:537541

Sato T, Haneda K, Seki M, Iijima T (1990) Morphology and magnetic properties of ultrafine $\mathrm{ZnFe}_{2} \mathrm{O}_{4}$ particles. Appl Phys A50:13-16
Smit J, Wijn HPJ (1959) Ferrites. Philips' Technical Library, Eindhoven

Zabukovec Logar N, Novak Tušar N, Mali G, Mazaj M, Arčon I, Arčon D, Rečnik A, Ristić A, Kaučič V (2006) Manganesemodified hexagonal mesoporous aluminophosphate MnHMA: synthesis and characterization. Microporous Mesoporous Mater 96:386-395 